This is the peer reviewed version of the following article: Jurcik, T., Yakobov, E., Solopieieva-Jurcikova, L., Ahmed, R., Sunohara, M., \&; Ryder, A. G. (2015). Unraveling ethnic density effects, acculturation, and adjustment: The case of Russian-speaking immigrants from the former Soviet Union. Journal of Community Psychology, 43(5), 628-648. doi:10.1002/jcop.21708, which has been published in final form at https://doi.org/10.1002/jcop.21708. This article may be used for non-commercial purposes in accordance with Wiley Terms and Conditions for Use of Self-Archived Versions.

\title{
Unraveling Ethnic Density Effects, Acculturation, and Adjustment: The Case of Russian-Speaking Immigrants from the Former Soviet Union
}

\author{
Tomas Jurcik $^{1}$, Esther Yakobov ${ }^{1}$, Liza Solopieieva-Jurcikova ${ }^{1}$, Rana Ahmed $^{1}$, Momoka Sunohara ${ }^{2}$, and \\ Andrew G. Ryder ${ }^{1,3}$ \\ ${ }^{1}$ Concordia University \\ ${ }^{2}$ McGill University \\ ${ }^{3}$ Jewish General Hospital
}

\begin{abstract}
There has been limited advancement in the empirical literature unpacking the ethnic density effect, a social ecological phenomenon that may help explain some of the conflicting findings in bidimensional acculturation research. In this study, we developed a brief measure of perceived ethnic density in a community sample of Russian-speaking immigrants $(N=269)$ in Montreal, Canada, finding it to be a superior predictor of distress to objective linguistic density. Acquiring social support partly mediated the relation between perceived ethnic density and lower distress. Furthermore, the relation between heritage acculturation and distress was double moderated by perceived ethnic density and time lived in the neighborhood. A person-ecology match involving heritage acculturation and ethnic density was related to better psychological adjustment for participants who had resided in their neighborhood for less than, but not more than, 2 years. Clinical and community research implications for using measures of perceived ethnic density and acculturation measurement are discussed.
\end{abstract}

A once common belief in North American social science was that "without understanding social structures, personal troubles cannot be solved" (Oishi et al., 2009, p.139). However, in recent decades the empirical literature in social and cultural psychology has been relatively devoid of contextualizing research in social-ecological systems, such as institutional structures and neighborhood environments. For instance, only limited empirical work has been conducted to examine how immigrant acculturation may interact with environmental contexts, despite a long and rich tradition of acculturation research in psychology and anthropology (e.g.,

The research described in this article was supported by an FQRSC doctoral award to Tomas Jurcik and an FRSQ chercheurboursier award to Andrew G. Ryder. This research was conducted as part of Tomas Jurcik's doctoral dissertation at Concordia University, supervised by Andrew G. Ryder.

We thank Tatiana Kruglikova, Gramota, and Mechta schools and various Montreal immigrant associations for their invaluable help with recruitment; Sarah Said, Tarek Haidar, and David-Alexandre Bosset for their research assistance; Drs. Edison Trickett and Laurence Kirmayer for pertinent references and inspiration; as well as members of the Culture, Health and Personality Lab at Concordia University for their general support.

Please address correspondence to: Tomas Jurcik and/or Andrew Ryder, 7141 Sherbrooke St. W., Montreal, Quebec, H4B 1R6, Canada; e-mail: t_jurcik@live.concordia.ca and/or andrew.ryder@concordia.ca
Berry, 2006a, 2006b; Redfield et al., 1936).

There has also been a burgeoning of a parallel epidemiological literature since the 1930s investigating the ethnic density (ED) effect-the phenomenon that minority groups often have better adjustment outcomes if they live in areas with a greater proportion of people of the same ethnicity (e.g., Das-Munshi et al., 2012; Faris \& Dunham, 1939). Although the acculturation and ethnic density literatures rarely intersect or inform one another (for recent exceptions, see: Jurcik, Ahmed, et al., 2013; Kwag et al., 2012; Miller et al., 2009), a collaboration between researchers that focus on social ecology and culture might shed light on the inconsistencies permeating acculturation research (e.g., Trickett et al., 2009) and on clarifying the mechanisms of the ED effect (see Shaw et al., 2012). Community psychology, with its ecological focus, may provide acculturation research with a missing contextual link (Trickett et al., 2009).

As an extension of a preliminary study on immigrant students (Jurcik, Ahmed, et al., 2013), the current research attempts to further bridge this gap by exploring the effects of acculturation and perceived ethnic density on adjustment in Russian-speaking immigrants from the Former Soviet Union (FSU), a traditionally understudied but growing minority group in North America. We will begin, however, by separately exploring some of the gaps in acculturation and ethnic density research and then examine how the two literatures might be linked to fill these lacunae. 


\section{Acculturation Research: What Is It Good For?}

There have been numerous inconsistencies across studies in operational conceptualizations of acculturation, fueling the contentiousness of theoretically and empirically informed debates on the topic (e.g., Rudmin, 2009; Schwartz et al., 2010; Trickett et al., 2009). The more simple unidimensional models, which view acculturation as assimilation to the mainstream cultural context, have been found to lack explanatory power compared to bidimensional models (e.g., Ryder et al., 2000; Schwartz et al., 2010). The latter sort posit two conceptually independent dimensions, construing acculturation as the degree to which migrants adapt or maintain the beliefs, values, and behaviors associated with both mainstream and heritage cultural contexts. Additional dimensions may emerge-for example, a third or fourth cultural affiliation (e.g., Persky \& Birman, 2005)-although less research has been conducted with more than two dimensions.

Extensive research has demonstrated how different acculturation styles or "strategies" are related to mental health outcomes, an important question for policy makers and clinicians alike (Berry, 2006b; Sam, 2006). Berry's (e.g., 2006b) well-known approach suggests that integration of both mainstream and heritage cultural self-identities is the most psychologically adaptive, while the most problematic reflects marginalization from both cultural self-identities. Those who engage in separation (away from the mainstream cultural context) and assimilation (towards the mainstream cultural context) strategies fall between these two extremes. However, the evidence is not always so clear-cut: under some environmental and psychological conditions, one type of acculturation style may be more adaptive than another type, leading some to argue that the term "strategies" is misleading by overemphasizing personal choice at the expense of contextual influences (Schwartz et al., 2010). For instance, in Vancouver, the adjustment of minority students was dependent on mainstream but not heritage acculturation (Ryder et al., 2000; a similar effect was found in Germany: Zhang et al., 2010).

Contrast this with Montreal, the setting of the current study, where heritage-but not mainstream-acculturation played a role as a predictor or moderated predictor in the adjustment outcomes of a diverse group of immigrants (Ahmed et al., 2011; Jurcik, Ahmed, et al., 2013). With its two mainstream cultural groups (i.e., English Canadian and French Canadian) and greater segregation, Montreal may foster the salience of heritage culture affiliations compared to Vancouver (see Balakrishnan et al., 2005). In other studies, even outright rejection of a particular cultural identification may be plausibly adaptive (e.g., Schwartz et al., 2010). For example, Muslim participants in Toronto were at increased risk of depressive symptoms when identifying with the mainstream culture, but were protected through their heritage culture affiliations (Asvat \& Malcarne, 2008).
The lack of a clear message in the acculturation literature has proven less than useful on the front lines. Some community psychologists have referred to the acculturation literature as "confusing, contradictory, and non-cumulative" (Trickett et al., 2009, p.268). Even less flattering, Landrine and Klonoff (2004, p.530) argue that the "evidence is so incoherent that it is unintelligible, and hence it continues to be largely useless to health psychology and behavioral medicine." Given that acculturation research is often decontextualized (Trickett et al., 2009), a potential solution would be to gather evidence about moderating factors, which may lead us towards a better understanding of if, and when, acculturation plays a predictive role. Perhaps sociopolitical climate at the macro level (e.g., Berry, 2006a) as well as city and neighborhood environments at lower levels (e.g., Jurcik, Ahmed, et al., 2013) shape, favor, or demand some acculturation styles over others.

As a neighborhood variable, ED or immigrant concentration has been recently studied as a moderator of the effects of acculturation on psychological adjustment (Jurcik, Ahmed, et al., 2013; Kwag et al., 2012; Miller et al., 2009). This contextual variable among others such as social capital and neighborhood deprivation, may help researchers understand the disparate findings within the acculturation literature.

\section{Making Sense of Ethnic Density}

Psychologists have traditionally neglected social contextual factors in mental health, but this trend appears to be reversing in recent years (e.g., Juang \& Alvarez, 2011; Jurcik, Ahmed, et al., 2013; Miller et al., 2009; Syed \& Juan, 2012). For considerably longer periods, epidemiologists in social psychiatry have made important advances in showing the relevance of local area contexts (e.g., Bécares et al., 2009; DasMunshi et al., 2010; Das-Munshi et al., 2012; Shaw et al., 2012). The ethnic density effect is one such variable-living in a neighborhood with a greater proportion of people from the same ethnic group is generally associated with lower levels of physical or mental health problems. Faris and Dunham (1939) originally demonstrated this effect when they examined psychiatric hospitalizations in Black and White patients depending on neighborhood composition in Chicago. Since that time, ED has been studied in various groups and settings and has been found to be protective against physical problems, psychosis, and common mental disorders and symptoms (e.g., Bécares et al., 2009; Bécares et al., 2012; DasMunshi et al., 2010; Das-Munshi et al., 2012; Jurcik, Ahmed, et al., 2013; Shaw et al., 2012).

ED can be measured objectively as well as subjectively and the two indices are moderately correlated (Juang \& Alvarez, 2011; Stafford et al., 2009). Much less research has been conducted with perceived ED until recently (e.g., Juang \& Alvarez, 2011; Jurcik, Ahmed, et al., 2013; Kwag et al., 2012; Stafford et al., 2009; Syed \& Juan, 2012). Given that 
perceptions of socioeconomic and environmental states tend to correlate with mental health outcomes better than objective ones (e.g., Christie-Mizell et al., 2003; Singh-Manoux et al., 2005), there is reason to believe that perceived ED will also be more strongly associated with other subjective experiences empirically.

The literature has not always been consistent in demonstrating the benefits of ethnic density, however. In some cases, a higher likelihood of negative health outcomes has been found. Thus, ED was detrimental for Pakistani, but not Indian or Bangladeshi, participants with respect to psychosis in the United Kingdom (Bécares et al., 2009). A recent review showed that although the majority of studies on mental disorders have obtained a protective effect, a number of neutral findings (i.e., no effect) have also been obtained, along with a smaller proportion of studies demonstrating a reverse effect, for example, with some visible minority adolescent samples (Shaw et al., 2012).

While the mechanisms of ED effect remain unexplained, researchers have recently suggested that social support and discrimination might mediate ED, and that the latter moderates discrimination and social support (e.g., Bécares et al., 2009; Das-Munshi et al., 2012; Jurcik, Ahmed, et al., 2013; Syed \& Juan, 2012; Whitley et al., 2006). Thus, ED has been hypothesized to offer a "psychic shelter" for immigrants (Whitley et al., 2006, p.389). Increased social support and decreased discrimination in ethnically dense areas (measured objectively) have indeed been shown to be protective for various South Asian ethnic groups in the United Kingdom against psychosis (Das-Munshi et al., 2012). ED has also moderated the effect of racism on physical health outcomes (Bécares et al., 2009).

In Montreal, a perceived ED effect for depression was shown to operate via lower discrimination (but not more likely social support) in a heterogeneous group of immigrant students, many of whom had visible minority backgrounds (Jurcik, Ahmed, et al., 2013; for contrasting see Birman et al., 2005; Juang and Alvarez, 2011). The Montreal study may be the only one to date to statistically demonstrate an indirect effect of discrimination between perceived ED and depression. Researchers continue mediating in their attempts to identify potential mediating mechanisms.

\section{Ethnic Density Meets Acculturation: Favoring a Good Match}

While the mechanisms of the ED effect remain to be clarified, ethnic density may help contextualize acculturation processes. Acculturation does not take place in a vacuum - the process and effects of acculturation are probably ecology-dependent. For instance, the acculturation patterns for FSU émigrés varied in two U.S. counties with differing levels of ED (Birman et al., 2005). For a group of women from the FSU, behaviors and practices related to Russian heritage were associated with greater alienation from American culture, but this effect was mitigated when living in areas with high (nonspecific) immigrant concentration (Miller et al., 2009). In another study, older Hispanic adults were more prone to depressive symptoms if they were not affiliated with the mainstream culture; moreover, depressive symptoms were further aggravated in ethnically dispersed neighborhoods (Kwag et al., 2012). Thus, ethnic density may act as a moderator of the acculturation-adjustment link according to the emerging evidence.

More recently in our pilot project with immigrant students, we found that heritage, not mainstream, acculturation was associated with less depression in Montreal, yet only for those who lived in neighborhoods with high perceived ED (Jurcik, Chentsova-Dutton, et al., 2013). This pattern is consistent with an ecology-acculturation fit or "match" model (Jurcik, Ahmed, et al., 2013). That is, those who match on heritage acculturation and neighborhood ED (high-high or low-low) have a mental health advantage over those who mismatch (low-high or high-low). Similar findings have suggested that a fit between family and personal heritage acculturation is better for mental health than a mismatch (Asvat \& Malcarne, 2008). Person-environment mechanisms may thus help account for some of the discrepancies in the literature reviewing ED effects (see Shaw et al., 2012).

A further overlooked variable that may moderate the ED effect itself is the length of time lived in a neighborhood. The perceived benefits of a neighborhood environment likely take time to establish (e.g., social cohesion). Thus, very recent arrivals to a local area may have had less opportunity to benefit from the effect than more established residents. We are not aware of published research to date exploring such a moderating effect.

In sum, acculturation needs to be examined in the context of ecological systems to improve the coherence of research findings. The inconsistencies in the ethnic density and acculturation literatures will likely be difficult to resolve without accounting for both variables simultaneously. Understanding contextual considerations may be useful to clinicians and policy makers who need to know under what circumstances (i.e., when, where, and with whom) particular dimensions of acculturation may play a protective-or detrimental-role.

\section{The Adjustment of Russian-Speaking Immigrants in Montreal}

There has been an influx of Russian-speaking immigrants since the collapse of the former Soviet Union to North America and other Western countries (e.g., Mirsky, 2009). In Montreal alone, the population of Russian speakers has increased by almost $29.5 \%$ between 2006 and 2011 while the overall population of the city grew by about $5.2 \%$ (see Statistics Canada, 2006, 2011). Despite these striking demographic shifts, Russian immigrants are relatively neglected 
in cultural, community, and clinical research (e.g., Hundley \& Lambie, 2007; Jurcik, Chentsova-Dutton, et al., 2013). This relatively educated immigrant group is not immune to acculturative stress and adaptation difficulties, however (e.g., see Jones \& Trickett, 2005; Jurcik, Chentsova-Dutton, et al., 2013, for review).

Additionally, this population is of considerable theoretical interest because it primarily represents a nonvisible minority group and may be distinct from East Asian cultural groups that have been more commonly studied, as well as Euro-American samples (see Jurcik, Chentsova-Dutton, et al., 2013). For instance, rather than supporting autonomy, Russians tend to emphasize direct and unsolicited instrumental (or "in-your-face") social support more so than EuroAmericans (Chentsova-Dutton \& Vaughn, 2012, p.690). Often migrating as families, findings demonstrate that Russians who enjoy social (including family) support are protected against depression (Mirsky, 2009).

While our previous study on ED and acculturation was limited by its highly heterogeneous sample composition (Jurcik, Ahmed, et al., 2013), our focus here on Russian-speakers allows us to study a group that shares a common language, history, practices, and meanings despite its diversity (see Jurcik, Chentsova-Dutton, et al., 2013, for review). We thus determined that Russian speaking immigrants to Montreal were good candidates for helping us understand the interrelation of acculturation and ED.

\section{Aims and Hypotheses}

The aim of the current study is three-fold. First, we hope to extend our previous findings (Jurcik, Ahmed, et al., 2013) by clarifying the mediating mechanisms of the perceived ED effect in a more homogeneous sample of Russian speaking immigrants. Second, we will examine whether both subjective and objective ED, as well as length of neighborhood residence can contextualize acculturation-adjustment outcomes. Third, we will test whether a brief composite measure of perceived ED is correlated with an objective indicator.

\section{Correlational hypotheses}

These were developed in preparation for the mediation analysis and in order to validate the composite subjective ED measure against objective ED. Perceived ED will thus be negatively associated with symptoms (as measured by distress and depression) and discrimination, and positively with social support (as measured by two social support scales); in turn, social support will be negatively associated with distress and discrimination will be positively associated with distress. We also anticipate that objective linguistic density (proxy for objective ED) will show a similar pattern, albeit with weaker effect sizes, and be related to subjective ED.

\section{Indirect effect hypotheses}

The negative association between perceived ED and distress will be mediated by decreased discrimination and increased social support.

\section{Moderation hypotheses}

The association between heritage acculturation and distress will depend on levels of perceived ethnic density. Thus, an acculturation-ecology match between perceived ED and heritage acculturation (i.e., high on both, or low on both, variables) will be associated with less distress than a mismatch (e.g., high-low). We also expected for the protective association between ED and distress to be amplified by years resided in the neighborhood. Moreover, we examined a potential three-way interaction between heritage acculturation, $\mathrm{ED}$, and years resided in the neighborhood.

Additional exploratory models will examine (a) the moderation hypotheses above by replacing heritage with mainstream acculturation (e.g., Kwag et al., 2012) and (b) whether ED moderates effects of social support and discrimination (e.g., Bécares et al., 2009). Finally, perceived ED will be replaced with objective linguistic density in the final set of exploratory analyses retesting the moderation hypotheses described above.

\section{Method}

\section{Participants}

The final sample comprised 269 participants. Demographic information is provided in Table 1 . The mean age was 34.2 (standard deviation $[\mathrm{SD}]=7.70$ ), and $68 \%$ of the sample was female. Most participants were immigrants from Russia or Ukraine $(60 \%)$. Participants had lived in Canada for a mean of about 5 years, and a vast majority of participants lived with someone $(89 \%)$, generally family. Most of the participants found out about the study through online sources $(72.5 \%)$ and chose to complete the survey in Russian $(77 \%)$ rather than English $(23 \%)$. Participants were retained if they were Russian speaking and born in the FSU, migrants to Canada currently living in Montreal, and if they indicated that they responded honestly to the survey questions.

\section{Procedure}

Immigrants living in Montreal completed an extensive online survey between 2011 and 2012 on the psychosocial adjustment to life in Canada (the current study analyzed a subset of the measures). We oversampled Russian speakers in the community by making the study available in Russian and English languages, and by advertising in both languages through various online (e.g., Craigslist, Russian forums) and print media sources (i.e., a local Russian newspaper) as well as immigrant community centers and the parents of children 
Table 1

Demographic Variables for Immigrants From Former Soviet Union to Montreal, Canada $(N=269)$

\begin{tabular}{|c|c|c|}
\hline \multicolumn{3}{|l|}{ Variables } \\
\hline Gender (\%female) & 68.0 & \\
\hline Age (years): Mean (SD) & 34.16 & $(7.71)$ \\
\hline Canada (years): Mean (SD) & 5.05 & $(5.24)$ \\
\hline Quebec (years): Mean (SD) & 4.81 & $(5.17)$ \\
\hline \multicolumn{3}{|l|}{ Country of birth $(\%)$} \\
\hline Russia & 34.7 & \\
\hline Ukraine & 25.4 & \\
\hline Moldova & 15.3 & \\
\hline Belarus & 9.0 & \\
\hline Kazakhstan & 5.6 & \\
\hline Other Former Soviety Union & 10.0 & \\
\hline \multicolumn{3}{|l|}{ Language of survey $(\%)$} \\
\hline Russian language survey & 77.0 & \\
\hline English language survey & 23.0 & \\
\hline \multicolumn{3}{|l|}{ Referrals $(\%)$} \\
\hline Russian Forum & 40.4 & \\
\hline Online Advertisement & 39.2 & \\
\hline School & 12.7 & \\
\hline Other (friend, family, newspaper, etc.) & 7.7 & \\
\hline
\end{tabular}

Note. Immigrants born outside of the FSU were excluded. $N=269$.

attending Russian schools; others had been informed about the research via personal acquaintances of the researchers (see Table 1 for referral source). To promote snowball sampling, participants were encouraged to inform acquaintances and family; however, only one participant per household was permitted to participate. The study was confidential and participants consented to the study online.

Instruments were translated into Russian by an experienced translator (L.S.-J.) and verified by two native speakers (including E.Y.). Measures that had been obtained from existing sources were also verified. Participants had the option of completing the survey in Russian or in English and entered a raffle for five prizes of $\$ 100$, which was drawn after completion of the study. The university's institutional review board approved the research.

\section{Measures}

Alphas are presented as a whole collapsed across language versions. Following some basic demographic questions, the participants proceeded to the measures.

Perceived Ethnic Density-Composite (Perceived ED; extension of Stafford et al., 2009)

Perceived ED was measured using a item-item scale to estimate the participants' neighborhood ethnic concentration. Participants were asked to think of their local area (15-20 minutes walking distance from their home), and for the first item, to estimate "what proportion of all the people in this local area are of the same ethnic group" as the participant on a 5-point scale, ranging from 0 (none or hardly any) to 4 (almost all or all of the local area). Three additional items were developed, asking participants to what extent in their local area they had access to ethnic "specialty products" such as food and "resources and organizations" such as community centers related to their ethnic group, and whether they could get by and be understood in their native language in their area. Also on a 5-point scale, these items ranged from 0 (not at all) to 4 (a large extent). Higher mean item scores for the four items combined reflected greater overall perceived ED. The scale was found to have adequate internal consistency ( $\alpha$ $=.73)$.

\section{Objective ethnic density}

Russian linguistic density was the proxy used for objective ED and was calculated at the level of the Forward Sortation Area (FSA; the first three digits of Canadian postal codes), which represent relatively small neighborhood districts. Census data (Statistics Canada, 2011) were used to determine the proportion of inhabitants who declared Russian to be their mother tongue (numerator) to the total number of inhabitants (denominator) within the FSA. Although the range was low (.00 to .08), objective linguistic density was found to be positively correlated with perceived ED, $r(247)=.46, p<.001$.

\section{Vancouver Index of Acculturation (VIA; adapted from Ry- der et al., 2000)}

The adapted VIA assesses heritage and mainstream acculturation (i.e., French Canadian and English Canadian) on three independent scales. Each scale comprises 10 items measuring identical experiences on a 9-point Likert scale ranging from 1 (strongly disagree) to 9 (strongly agree). Heritage and mainstream subscales are considered to be conceptually and empirically orthogonal (Ryder et al., 2000). A sample heritage item is "I enjoy the jokes and humor of my heritage culture," and a sample mainstream item is "I believe in mainstream English-Canadian values." To obtain a single measure of mainstream acculturation, the highest item scores for either French or English mainstream acculturation were retained. Heritage ( $\alpha=.84)$ and mainstream acculturation $(\alpha$ $=.85$ for both English and French) subscale scores showed good internal reliability.

\section{Multidimensional Scale of Perceived Social Support (MSPSS; Zimet et al., 1988)}

The MSPSS is a 12-item measure assessing general subjective social support in the domain of family, friends, and significant others on a 7-point Likert scale ranging from 1 (very strongly disagree) to 7 (very strongly agree). A sample item is "I can talk about my problems with my friends." It 
was used to explore the association between social support and depression in a previous study with Russian immigrants (Ritsner et al., 1997). Internal reliability was excellent for the current sample $(\alpha=.93)$.

\section{Family Crisis Oriented Personal Scales (F-COPES; Mc- Cubbin et al., 1996)}

The F-COPES comprises 29 items that explore effective family coping strategies related to difficulties in five domains: Acquiring Social Support (AcSS; nine items, $\alpha=$ .79); Mobilizing the Family to Accept Help (MFAM; four items; $\alpha=.84$ ); Seeking Spiritual Support (SPIRIT; four items, $\alpha=.84$ ); Reframing (REFR; eight items, $\alpha=.76$ ); and Passive Appraisal (PA; four items, $\alpha=.63$ ). The first three represent an active, external coping factor and the second two an internal factor. The scale uses a 5-point Likert scale ranging from 1(strongly disagree) to 5 (strongly agree).

Sample items from the AcSS domain are "sharing concerns with close friends" and "receiving gifts and favors from neighbors." Items in the SPIRIT domain were modified slightly to be more encompassing because the original scale assumed a Christian belief system. "Participating in religious/spiritual activities" is an item from this domain. A sample item for MFAM is "seeking assistance from community agencies and programs designed to help families in our situation." Sample items for PA and REFR respectively are "watching television" and "showing that we are strong."

\section{Perceived Discrimination Scale (PERDS; Noh E Kaspar, 2003)}

The PERDS is an eight-item measure used to assess subjective experiences related to discrimination in Canada on a 5-point scale ranging from 1 (Never) to 5 (All the Time). A sample item is "In Canada, because of discrimination, have you ever been treated unfairly?" The measure has been associated with depression in Korean immigrants (Noh \& Kaspar, 2003) and in the current study it showed excellent internal reliability $(\alpha=.93)$.

\section{General Health Questionnaire (goldberg1992)}

The GHQ-12 assesses general psychiatric morbidity using 12-items on a 4-point scale ranging from 0 (not at all) to 3 (much more than usual) that are related to a broad range of psychiatric symptoms in the past 2 weeks (e.g., lost much sleep over worry, been losing confidence in yourself). Internal reliability was adequate in the current sample $(\alpha=.78)$. A Russian version of the scale was used (Ponizovsky et al., 2007).

\section{Center for Epidemiologic Studies-Depression (CES-D; Radloff, 1977)}

The CES-D is a 20-item scale assessing depressive symptoms over the last week on a 4-point scale ranging from 0 (rarely or never) to 3 (most of the time) and has been used in Russia (Dershem et al., 1996). More specific than the GHQ, examples of items include poor appetite, sadness, and loneliness. Internal reliability was excellent for the current sample $(\alpha=.91)$.

\section{Design and Analyses}

A listwise correlation matrix was examined for the first set of hypotheses, along with a general linear model (GLM) to test whether ED was a significant overall predictor of distress (CES-D and GHQ) and social support (MSPSS and AcSS). Bootstrapping analysis (with 5,000 resamples; Hayes, 2013; Preacher \& Hayes, 2008) was used to test for mediation related to the second hypotheses, and hierarchical multiple regression was used to test for the final moderation (interaction) hypotheses and three-way test: here ED was dichotomized (high vs. low) along with years lived in the neighborhood (less than 2 years vs. more than 2 years). Post hoc analyses probing distress in different subgroups of ethnic density and length of neighborhood residence controlled for alpha inflation using Tukey's HSD. Exploratory moderation tests (e.g., using objective instead of perceived ED, and discrimination and social support instead of acculturation) were also conducted.

\section{Results}

All analyses were conducted in SPSS version 20 (IBM Corp, 2011) and mediation analyses were conducted using PROCESS (Hayes, 2013), an SPSS macro. PROCESS uses bootstrapping, a statistical method that is more robust with samples that may not meet the standard assumptions of inferential statistics (Preacher \& Hayes, 2008). Univariate outliers were winsorized to 3.3 standard deviations from the mean with rank order preserved. Three multivariate outliers were identified using the Mahalanobis distance cutoff, $\chi^{2}(13)=34.53, p<.001$, and were deleted. Missing data for the items related to the measures in the correlation table and years of neighborhood residency was not extensive (between 0 and $2.6 \%$ for items) and completely at random, having passed Little's MCAR test, $\chi^{2}(7833)=7916.17, p=$ .25. Imputations were not conducted but mean item scores were calculated in the syntax for participants who completed at least two thirds of each multi-item measure. Listwise Ns are reported for the analyses related to the hypotheses.

For the initial hypotheses, the zero-order correlation matrix (see Table 2) revealed numerous small effects associated with perceived $\mathrm{ED}$ (composite). ED was a significant predictor of reduced general distress and greater problem-focused social support, although ED was not significantly related to depression, general social support, or discrimination. Both general and acquired social support were related to less distress, while discrimination was positively related to depression but not general distress. ED was also significantly re- 
lated to the other external patterns of F-COPES, seeking spir-

itual support and mobilizing family, but not to the internal patterns of passive acceptance and reframing. Objective linguistic density did not yield correlations other than with perceived ED (see measures), $r(247)=.46, p<.001$, and was not included in the table.

Two Multivariate GLMs were conducted to examine whether perceived ED could predict overall symptoms (GHQ-12 and CES-D) and overall social support (AcSS and MSPSS). ED was revealed to be a significant multivariate predictor for overall social support, $F(2,262)=3.50, p=.03$, Wilks' $\lambda=.974, \eta_{p}{ }^{2}=.026$; however, it was only a significant univariate predictor of F-COPES-AcSS, $F(1,263)=$ $6.95, \eta_{p}{ }^{2}=.026, p=.01$, and not MSPSS, $F(1,263)=1.32, p$ $=.25$. Similarly, ED showed a significant multivariate effect for psychological distress, $F(2,265)=3.31, p=.04$, Wilks' $\lambda=.976, \eta_{p}{ }^{2}=.024$, but ED was only a univariate predictor for GHQ-12, $F(1,266)=6.43, p=.01, \eta_{p}{ }^{2}=.024$, and not CES-D, $F(1,266)=1.42, p=.23$. It was thus determined that it would be acceptable to use only GHQ-12 and F-COPES AcSS in the subsequent mediation and regression analyses. Age, gender, and household income were not correlated with GHQ-12; background variables were therefore not included as covariates in the mediation or moderation models.

For the second set of hypotheses, the bootstrapped mediation analysis (see Figure 1) indicated that the relation between perceived ED and distress was partly explained by a significant indirect effect of acquiring social support, as percentile-corrected 95\% confidence intervals (CI) did not include zero. Given that all F-COPES external coping variables (acquiring social support, spiritual support, mobilizing family) correlated with perceived ED and distress, we explored whether they could significantly mediate the perceived ethnic density effect overall (i.e., when collapsed into one factor). The indirect effect $(-.0258$, standard error [SE] $=-.0107)$ for this external coping model was also significant, percentile corrected 95\% CIs [-.0494, -.0076]. However, given the marginal increase in the indirect effect for the external factor over the simple mediation model of acquiring social support alone, the additional two variables (spiritual support, mobilizing family) did not display significant indirect effects in a multiple mediation model assessing the three external variables simultaneously and separately (i.e., in parallel).

To test the moderation hypotheses (see Table 3), a hierarchical multiple regression was conducted. Main effects were entered in Block 1 and two-way interaction terms were added in Block 2, and the three-way interaction in Block 3. To preserve adequate group sizes and better visualize interactions, perceived ED was collapsed into two approximately equal groups, low (mean item score of equal or less than $.25, n=$ 128 ) or high (mean item core of more than .25, $n=137$ ); sim- ilarly, the number of years lived in the neighborhood (originally an ordinal variable) was also split into approximately equal groups of low (less than 2 years, $n=145$, hereafter termed recent residents) or high (more than 2 years, $\mathrm{n}=120$, hereafter termed established residents).

Table 3 presents standardized beta $(\beta)$, semipartial correlations $(s r), t$ test $(t)$ and related probability $(p)$ values. The overall model was significant, $F(7,257)=2.26, p=$ .03 , and accounted for $5.8 \%$ of the variance (3.2\% adjusted). Here, the hypothesized two-way interactions, perceived ED by years resided in the neighborhood and ED by heritage acculturation, were only marginally significant. However, the ED by heritage acculturation interaction was significantly moderated by length of neighborhood residence in the third step. Portrayed in Figure 2, the ED by heritage acculturation interaction for recent residents is found in the left panel and for established residents in the right panel.

\section{Post Hoc and Further Exploratory Analyses}

Follow up one-way analyses of variance (ANOVAs) were conducted to determine whether the four subgroups treated as levels of an independent variable (recent residents: low $\mathrm{ED}, n=71$, and high ED, $n=74$; established residents: low $\mathrm{ED}, n=58$, and high ED, $n=63$ ) differed on any of the measures used in the zero-order correlation matrix (i.e., Table 2 measures treated as outcome variables). To limit Type I error, Tukey HSD tests were examined following a significant result on inspection of one-way between subjects ANOVA (see Table 4). For four of the six significant comparisons obtained (all $p \mathrm{~s}<.05$ ), the low ED group for established residents was different from another group.

For GHQ-12 symptoms, the low ED established residents had higher scores than the high ED established residents. Analogously, the low ED established residents also appeared more symptomatic on the CES-D than the high ED established residents. The low ED established residents also reported more discrimination than the low ED recent residents, as well as less general social support (MSPSS) than the latter. In sum, the low ED established residents appeared to show a pattern of greater vulnerability.

Despite being more symptomatic, the downward slope in Figure 2 (right panel) suggested that heritage acculturation protected the low ED established residents. Exploratory moderation analyses confirmed that heritage acculturation buffered the effects of low social support, $R_{c h}^{2}=.09, F(1,53)$ $=7.06, p=.01$, and marginally buffered the effects of discrimination, $R_{c h}^{2}=.06, F(1,53)=3.63, p=.06$, on distress. This interaction did not hold for the other three subgroups.

Analogous exploratory two- and three-way ED moderation analyses to those presented in Table 3 were probed first by replacing heritage with mainstream acculturation (VIA$\mathrm{M})$, then social support (F-COPES and MSPSS), and finally discrimination (PERDS). None of these yielded significant 
JURCIK ET AL.

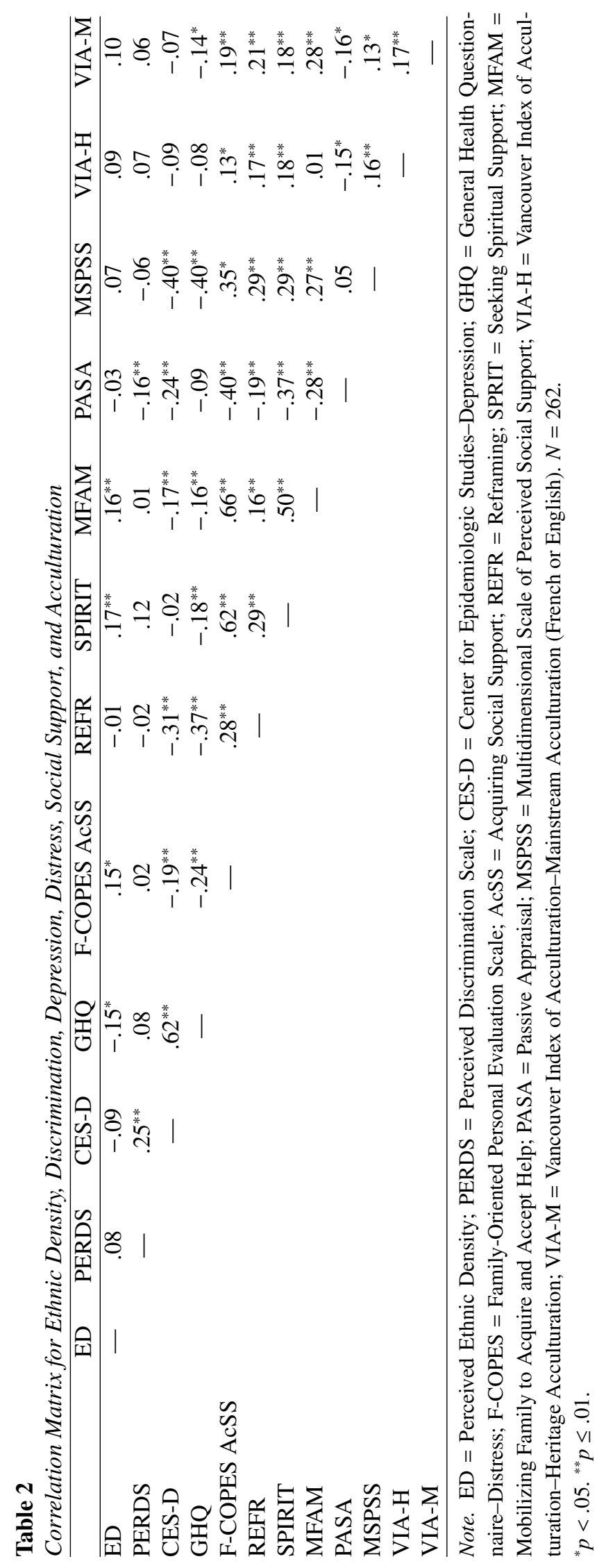




\section{Figure 1}

The perceived ethnic density and distress relation mediated by acquiring social support, using bootstrapping analysis

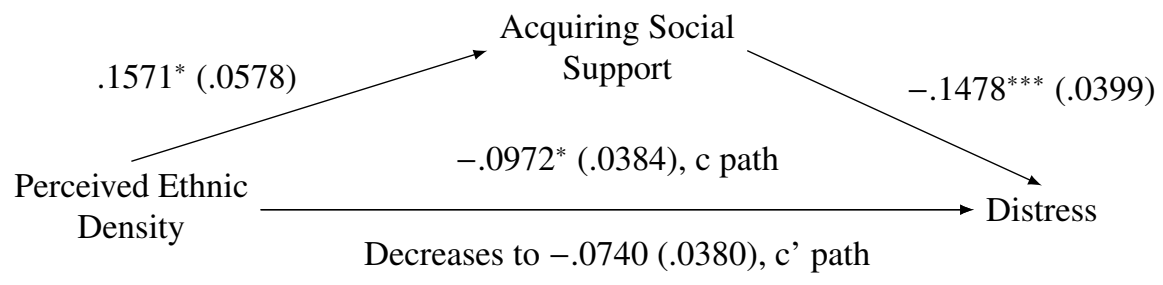

Note. Indirect effect $=-.0232, \mathrm{SE}=.0103$, Percentile corrected 95\% CI $[-.0457,-.0056]$ did not include zero. A bootstrap test with 5,000 resamples was used (Hayes, 2013). $N=266 .{ }^{*} \mathrm{p}<.05 .{ }^{* * *} \mathrm{p}<.001$.

Table 3

Hierarchical Multiple Regression of Distress (GHQ-12) on Ethnic Density, Acculturation, Years Lived in Neighborhood, and Interactions

\begin{tabular}{|c|c|c|c|c|}
\hline \multirow[b]{2}{*}{ Variables } & \multicolumn{4}{|c|}{ Final step } \\
\hline & $\beta$ & $s r$ & $t$ & $\bar{p}$ \\
\hline \multicolumn{5}{|l|}{ Main effects (Block 1) } \\
\hline${ }^{\text {a} E t h n i c ~ d e n s i t y ~(E D) ~}$ & -.02 & -.01 & -.22 & .83 \\
\hline${ }^{\text {a }}$ Time in neighborhood & .06 & .04 & .62 & .54 \\
\hline Heritage acculturation (VIA-H) & .09 & .05 & .83 & .41 \\
\hline \multicolumn{5}{|l|}{ Ethnic density interactions (Block 2) } \\
\hline${ }^{\mathrm{a}} \mathrm{ED} \times \mathrm{X}$ VIA-H & -22 & -.11 & -1.84 & .07 \\
\hline${ }^{a}$ ED x time (Neighborhood) & -.20 & -.12 & -1.93 & .06 \\
\hline${ }^{\text {a}}$ VIA-H x time (Neighborhood) & -.22 & -.11 & -1.84 & .07 \\
\hline \multicolumn{5}{|l|}{ Time on neighborhood interactions (Block 3) } \\
\hline${ }^{\mathrm{a}}$ ED x VIA-H x time (Neighborhood) & .28 & .13 & 2.21 & .03 \\
\hline$R^{2}$ & .06 & & & \\
\hline$R^{2}$ Change & .02 & & & \\
\hline
\end{tabular}

Note. ED = ethnic density; VIA-H = Vancouver Index of Acculturation-Heritage Acculturation.

Standardized $(\beta)$ and semipartial correlations $(s r)$ are reported for the final step. $N=265$. Age, gender, and household income did not correlate with distress and were therefore not entered in step 0 (block 0 ). Italics indicate $p<.05$.

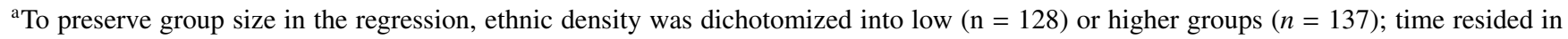
the neighborhood was also dichotomized into low (less than 2 years, $n=145$ ) or high (more than 2 years, $n=120$ ). 
Figure 2

Interaction between Ethnic Density, Heritage Acculturation, and Length of Neighborhood Residence for Psychological Distress

Less than 2 years of neighborhood residence

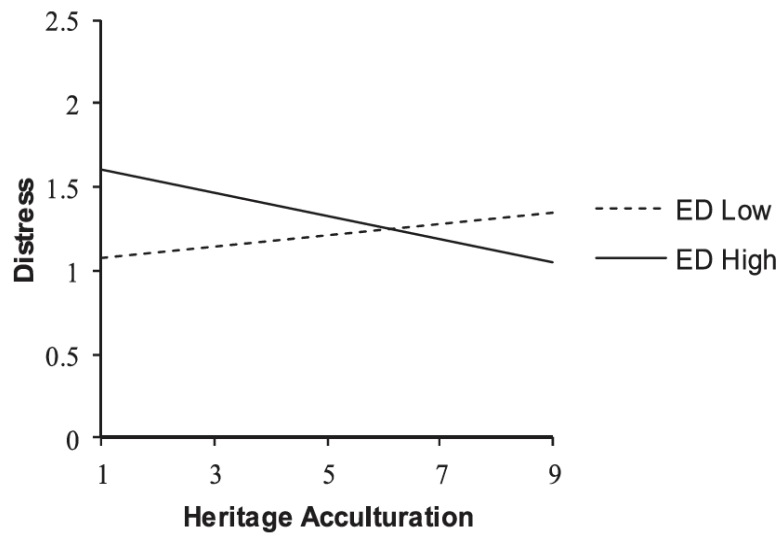

More than 2 years of neighborhood residence

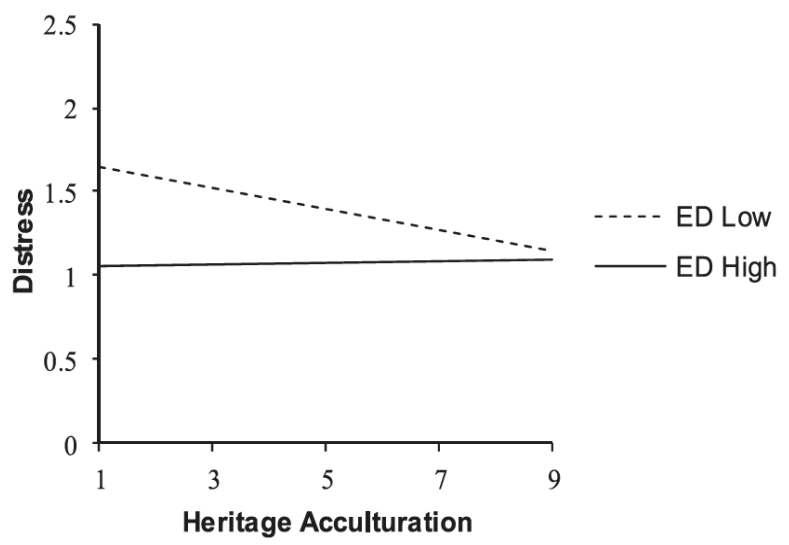

Note. Mean item scores for distress and heritage acculturation are presented without accounting for other variables in the model. The pattern for recent neighborhood residents (less than 2 years in the neighborhood) appears in the left panel and established residents (more than 2 years in the neighborhood) in the right panel.

Table 4

Differences in Distress, Depression, General Social Support, and Discrimination By Low and High Ethnic Density Groups That Are Recent or More Established in Their Neighborhood

\begin{tabular}{|c|c|c|c|c|c|c|c|c|c|c|c|c|}
\hline \multirow[b]{3}{*}{$\overline{\mathrm{GHQ}}$} & \multicolumn{4}{|c|}{ Recent } & \multicolumn{4}{|c|}{ Established } & \multicolumn{4}{|c|}{ ANOVA } \\
\hline & \multicolumn{2}{|c|}{ Low ED } & \multicolumn{2}{|c|}{ High ED } & \multicolumn{2}{|c|}{ Low ED } & \multicolumn{2}{|c|}{ High ED } & \multirow{2}{*}{$\frac{d f}{(3.262)}$} & \multirow{2}{*}{$\begin{array}{c}F \\
2.97\end{array}$} & \multirow{2}{*}{$\frac{\eta^{2}}{0.03}$} & \multirow{2}{*}{$\begin{array}{c}p \\
.032\end{array}$} \\
\hline & $1.23_{\mathrm{ab}}$ & $(0.45)$ & $1.22_{\mathrm{ab}}$ & $(0.45)$ & $1.29_{\mathrm{a}}$ & $(0.49)$ & $1.06_{b}$ & $(0.41)$ & & & & \\
\hline CES-D & $0.71_{\mathrm{ab}}$ & $(0.58)$ & $0.81_{\mathrm{a}}$ & $(0.53)$ & $0.93 \mathrm{a}$ & $(0.57)$ & $0.54_{b}$ & $(0.42)$ & $(3,261)$ & 5.94 & 0.06 & .001 \\
\hline MSPSS & $5.95_{\mathrm{a}}$ & $(0.96)$ & $5.69_{\mathrm{ab}}$ & (1.24) & $5.40_{\mathrm{b}}$ & (1.35) & $5.88_{\mathrm{ab}}$ & (1.04) & $(3,261)$ & 2.80 & 0.03 & .040 \\
\hline PERDS & $1.41_{\mathrm{a}}$ & $(0.51)$ & $1.73_{\mathrm{b}}$ & $(0.73)$ & $1.86_{\mathrm{b}}$ & $(0.76)$ & $1.65_{\mathrm{ab}}$ & $(0.63)$ & (3.261) & 5.49 & 0.06 & .001 \\
\hline
\end{tabular}

Note. $\mathrm{ANOVA}=$ analysis of variance $\mathrm{ED}=$ ethnic density; $\mathrm{df}=$ degree of freedom; GHQ = General Health Questionnaire; CESD = Center for Epidemiologic Studies-Depression; MSPSS = Multidimensional Scale of Perceived Social Support; PERDS = Perceived Discrimination Scale. Standard deviations appear in parentheses. Means sharing the same subscript letter do not differ significantly. Recent residents have lived in their neighborhood for less than 2 years, and established residents for more than 2 years.

interactions. Replacing perceived ethnic density with objective linguistic density in the models also did not yield significant moderation results. Since none of these exploratory interactions were significant, follow up comparisons and corrections for alpha inflation were not conducted.

\section{Discussion}

The current study aimed to unpack the ethnic density effect and assess acculturation in ecological context in first generation Russian-speaking immigrants from the FSU. The findings partly supported the hypotheses. For the first set, objective linguistic density and perceived ED were positively related, but as expected, perceived measurement was a better predictor in this small sample; the objective indicator did not correlate with any of the outcome variables. Thus, perceived ED was related positively to overall social support and was protective against overall psychological distress. However, although discrimination was a risk for distress, perceived ED was unrelated to perceived discrimination.

For the second set, the indirect effect hypothesis was partly supported: problem based acquired social support (but 
not general support or discrimination) mediated the relation between perceived ED and distress. Statistical findings related to the third set of hypotheses did not reach significance: the perceived ED effect was marginally amplified by length of neighborhood residence, and ethnic density marginally moderated the relation between heritage acculturation and distress.

More importantly, a significant three-way interaction qualified the marginal two-way interactions; a match between levels of ED and heritage acculturation (high-high or low-low), rather than a mismatch (e.g., high-low), was suggestive of less distress for participants who resided in the neighborhood for less than 2 years. This finding was consistent with our moderation hypothesis. However, at more than 2 years of residence, the pattern changed: those in lower ED neighborhoods showed more symptoms and psychosocial vulnerability (increased discrimination and less social support) relative to other groups, but appeared to benefit from heritage acculturation as a buffer. Objective ED and mainstream acculturation played no moderating role.

\section{Acculturation in Ecological Context}

In combination with our pilot study (Jurcik, Ahmed, et al., 2013), it appears that a fit or match between neighborhood ethnic ecology and heritage acculturation is predictive of better adjustment for diverse immigrant groups, at least at some points in time. Notably, we obtained an analogous crossover interaction pattern in two separate groups: a heterogeneous group of immigrant students (Jurcik, Ahmed, et al., 2013) and currently a community group of Russian-speaking immigrants. Obtaining similar findings with considerably different populations suggests the possibility of a universal phenomenon (Norenzayan \& Heine, 2005). However, this conclusion needs to be tempered by the three way interaction in the current study.

The current findings highlight some of the discrepancies in the ethnic density (e.g., Shaw et al., 2012) and acculturation literatures (e.g., Trickett et al., 2009). For instance, studies showing that minority adolescents may be placed at risk in ethnically dense neighborhoods (reviewed in Shaw et al., 2012) could potentially have been confounded by the possibility that younger immigrants may have had lower heritage culture affiliation compared to their parents (see also Asvat \& Malcarne, 2008). Using a similar logic, high heritage acculturation could have also been a risk factor in studies (e.g., Kennedy et al., 2005) for those persons who lived in ethnically sparse neighborhoods.

A mismatch between personal values (i.e., acculturation) and ecologies (i.e., neighborhoods) may thus create dissonance that manifests itself in psychological maladjustment (see Asvat \& Malcarne, 2008; Jurcik, Ahmed, et al., 2013). This mechanism could be present for more recent neighborhood residents when individuals may be especially sensitive to their environments (e.g., perhaps in transition periods as with recent immigrants and students). At later points, other mechanisms may become relevant. Groups living in less concentrated neighborhoods may become affected by general difficulties encountered by immigrants over time, such as accumulated discrimination experiences and difficulties in renewing social support, further aggravated in cases of limited heritage culture affiliation. Perhaps reflecting an unsuccessful assimilation attempt in individuals willing to shed their heritage culture by moving to lower ED neighborhoods, the findings imply a cumulative risk mechanism, possibly indicative of inadequate "psychic shelters" in such neighborhoods (see Whitley et al., 2006).

Although our study was not longitudinal and the interpretations offered here are speculative, our results strongly indicate that future research needs to explore ED effects in the context of not only acculturation, but also the length of neighborhood residency. Notably, mainstream acculturation played a modest role in the correlation matrix predicting adjustment, but it did not interact with perceived ED (as in our previous study, Jurcik, Ahmed, et al., 2013), suggesting that a match is more relevant with respect to heritage acculturation for improved adjustment. On the other hand, since Montreal represents a unique cultural and linguistic context, it is also possible that in other (e.g., less segregated) settings, ED may moderate the effects of mainstream acculturation (see Kwag et al., 2012).

\section{Unpacking Ethnic Density: One Size Does Not Fit All}

It is theoretically interesting that acquired (rather than general) social support played a mediating role in the current sample, suggesting that Russians may value practical and concrete support, especially when encountering problems (although we did not specifically measure imposed assistance; see Chentsova-Dutton \& Vaughn, 2012; Jurcik, Chentsova-Dutton, et al., 2013, for review). Such pragmatic support could potentially be harnessed in more concentrated areas, allowing Russians to benefit from this effect. Moreover, the ED effect may be partly explained by a combination of effective external coping mechanisms associated with family challenges (acquired social cohesiveness, mobilizing family to accept help and spiritual involvement), although only acquired social support was a significant mediating mechanism when considered separately. Perhaps in some minority cultural groups, local area ED effects operate mostly through friends, neighbors, and family in times of crisis. Instead, general social support (i.e., MSPSS) played no mediating role, and neither did decreased discrimination as in our previous study (Jurcik, Ahmed, et al., 2013).

In contrast, our previous sample contained a greater proportion of visible minority participants. Ethnic density has been inconsistently related to discrimination, sometimes more (Birman et al., 2005; Juang \& Alvarez, 2011) as well as 
less (Bécares et al., 2009; Das-Munshi et al., 2012), depending on group and setting. Perhaps ED does not operate via reduced discrimination in certain populations that are more likely to blend in with the mainstream; in this case, people of mostly Eastern European descent in a city with a strong European influence (Montreal) may not stand out in appearance and custom in contrast to other ethnic groups (Birman et al., 2005, for contrast, see).

There are likely other variables that play additional roles in explaining the ED effect (e.g., familiarity effects, enhanced cognitive efficiency during times of crisis). FSU migrants have also shown a tendency to preserve their cultural affiliations through Russian media and other sources (Kozulin \& Venge, 1995) and thus may benefit from ethnolinguistic vitality (Landry \& Bourhis, 1997). These findings and conjectures imply that ED mechanisms are not of the "one-size fits-all" variety, need to be culturally informed, and deserve further unpacking in future studies with various populations.

\section{Objective Versus Subjective Indicators}

Linguistic density (objective ED proxy) had no predictive role in the current sample, but this may be due to power issues that have plagued numerous studies (Shaw et al., 2012). On the other hand, our findings are in line with research that has shown that subjective social variables may be better predictors of experience than objective ones (ChristieMizell et al., 2003; Singh-Manoux et al., 2005, e.g., ), including a recent study that found subjective ethnic density to correlate with perceived availability of community resources (Juang \& Alvarez, 2011). Objective measures may thus be less connected to subjective experience, especially in smaller samples with limited power. It is also conceivable that perceived measurement may be more strongly associated with other subjective measures due to shared error variance. Nonetheless, the robust correlation which emerged between perceived and objective measurement indicated that our subjective measure had adequate construct validity.

\section{Limitations and Strengths}

The current study utilized a relatively small non-random sample, and methods of advertising (e.g., internet, reward) may have encouraged some members of the community to participate rather than others. For example, although there were multiple pathways to participation, the majority of the sample was female. Second, participants completed English or Russian versions of the survey that may have lacked complete equivalence, although internal consistency was good and we did not attempt to interpret mean differences across language versions. Third, we examined length of neighborhood residence, but because the study was cross-sectional, causality cannot be inferred. The study was also potentially prone to recall bias. Finally, we could have strengthened our cultural argument had we tapped into imposed or unsolicited support (e.g., Chentsova-Dutton, 2012).

In contrast, to our knowledge this is the first study to examine ethnic concentration and acculturation phenomena in Russian-speaking migrants in Montreal. Together our two studies (Jurcik, Ahmed, et al., 2013) demonstrate that ED effects likely operate through different and possibly selective mediators depending on group. Moreover, the current study adds to the mounting evidence that bidimensional measurement is likely to reveal findings obfuscated by unidimensional scales, but needs to be considered in ecological context (see Trickett et al., 2009). These results add to the growing psychological literature suggesting the importance of neighborhood contextualization in acculturation research (e.g., Birman et al., 2005; Jurcik, Ahmed, et al., 2013; Kwag et al., 2012; Miller et al., 2009).

\section{Future Directions: Community and Clinical Research Implications}

Longitudinal and mixed methods studies in multiple cities with various groups may generate new insights in unraveling ED effects. Moreover, little is known about the utility of ED in clinical settings for treatment planning (i.e., given the protective relation with psychosis and other mental disorders; Das-Munshi et al., 2010; Das-Munshi et al., 2012). Clinical researchers utilizing smaller samples may easily incorporate brief subjective measures of ED in routine assessments. The perceived ED findings, and their associated moderating effects, are small but deserve future attention. For example, Figure 2 (left panel) indicates approximately a .4-point difference on the GHQ-12 between high and low ED groups at lower levels of heritage acculturation. The mean item difference of .4 by 12 items represents about five points of the total score. The GHQ-12 threshold for caseness (i.e., presence of psychiatric morbidity) varies between studies, but is approximately three points (Goldberg et al., 1997, see). This indicates that some people may be especially sensitive to an ecology-acculturation match.

Our results also inform clinical findings from the therapist-client ethnic matching literature. Meta-analyses have shown negligible outcome differences for ethnic matching (e.g., Cabral \& Smith, 2011), but our findings and those of others (e.g., Asvat \& Malcarne, 2008) imply that perhaps a match/mismatch between heritage acculturation levels of both therapist and client could also be considered in predicting outcomes.

In terms of immigrant resettlement policy, our current and previous findings (Jurcik, Ahmed, et al., 2013) suggest that government agencies could study the effect of sensitizing immigrants (e.g., through brochures) to issues of ethnic density and heritage acculturation. Immigrant settlement choices that carefully consider these variables, along with other neighborhood and personal factors, might foster ad- 
justment through a person-environment fit. Issues of ED and matching aside, many other basic practical interventions related to stabilizing recent immigrants or refugees likely deserve priority, such as establishing safety and effective links with institutions and the mainstream society (e.g., Rousseau et al., 2011), as well as enhancing family based coping directly, as indicated in the current study.

\section{Conclusion}

There is much potential in reconsidering social ecological factors in the cultural and social psychological literatures. This study demonstrated that the relationship between heritage acculturation and psychological adjustment is complex but meaningful, and may depend on levels of ethnic density and length of neighborhood residence. Whereas a match between heritage acculturation and ethnic density may play a role at earlier phases of neighborhood residence, a different relation may operate for more longstanding residents. Our results also showed that the relation between higher perceived ethnic density and decreased psychological distress partly operated via acquiring social support during family difficulties. Future longitudinal research may attempt to replicate the current findings and shed light on the clinical utility of measuring perceived ethnic density and acculturation in clinically distressed immigrants. Because it has been difficult to resolve the acculturation debate without accounting for context, we hope that this work will further inspire researchers to consider a social ecological approach to acculturation.

\section{References}

Ahmed, R., Jurcik, T., \& Ryder, A. (2011). Acculturation and adjustment in visible minorities: An attachment perspective, Poster presented at the 72nd annual convention of the Canadian Psychological Association (CPA), Toronto, ON.

Asvat, Y., \& Malcarne, V. L. (2008). Acculturation and depressive symptoms in muslim university students: Personal-family acculturation match. International Journal of Psychology, 43(2), 114-124. https://doi. org/http://dx.doi.org/10.1002/andp.19053221004

Balakrishnan, T., Maxim, P., \& Jurdi, R. (2005). Social class versus cultural identity as factors in the residential segregation of ethnic groups in toronto, montreal and vancouver for 2001. Canadian Studies in Population [ARCHIVES], 203-227.

Bécares, L., Nazroo, J., \& Stafford, M. (2009). The buffering effects of ethnic density on experienced racism and health. Health $\mathcal{F}$ Place, 15(3), 700-708.
Bécares, L., Shaw, R., Nazroo, J., Stafford, M., Albor, C., Atkin, K., Kiernan, K., Wilkinson, R., \& Pickett, K. (2012). Ethnic density effects on physical morbidity, mortality, and health behaviors: A systematic review of the literature. American Journal of Public Health, 102(12), e33-e66.

Berry, J. W. (2006a). Contexts of acculturation. In J. W. Berry \& D. L. Sam (Eds.), The handbook of acculturation psychology (pp. 27-42). Cambridge University Press.

Berry, J. W. (2006b). Stress perspectives on acculturation. In J. W. Berry \& D. L. Sam (Eds.), The handbook of acculturation psychology (pp. 43-57). Cambridge University Press.

Birman, D., Trickett, E., \& Buchanan, R. M. (2005). A tale of two cities: Replication of a study on the acculturation and adaptation of immigrant adolescents from the former soviet union in a different community context. American Journal of Community Psychology, 35(1-2), 83-101. https :// doi .org/10 . 1007/ s10464-005-1891-y

Cabral, R. R., \& Smith, T. B. (2011). Racial/ethnic matching of clients and therapists in mental health services: A meta-analytic review of preferences, perceptions, and outcomes. Journal of Counseling Psychology, 58(4), 537. https://doi.org/10.1037/a0025266

Chentsova-Dutton, Y. E. (2012). Butting in vs. being a friend: Cultural differences and similarities in the evaluation of imposed social support. The Journal of Social Psychology, 152(4), 493-509. https://doi.org/ 10.1080/00224545.2011.642025

Chentsova-Dutton, Y. E., \& Vaughn, A. (2012). Let me tell you what to do: Cultural differences in advice-giving. Journal of Cross-Cultural Psychology, 43(5), 687-703. https :// doi . org/10 . 1177/ 0022022111402343

Christie-Mizell, C. A., Steelman, L. C., \& Stewart, J. (2003). Seeing their surroundings: The effects of neighborhood setting and race on maternal distress. Social Science Research, 32(3), 402-428. https://doi.org/ 10.1016/S0049-089X(03)00014-0

Das-Munshi, J., Becars, L., Dewey, M. E., Stansfeld, S. A., \& Prince, M. J. (2010). Understanding the effect of ethnic density on mental health: Multi-level investigation of survey data from england. British Medical Journal, 34, 1-9. https ://doi .org/10.1136/bmj . c5367

Das-Munshi, J., Bécares, L., Boydell, J. E., Dewey, M. E., Morgan, C., Stansfeld, S. A., \& Prince, M. J. (2012). Ethnic density as a buffer for psychotic experiences: Findings from a national survey (empiric). The British Journal of Psychiatry, 201(4), 282-290. https://doi.org/10.1192/bjp.bp.111.102376 
Dershem, L. D., Patsiorkovski, V. V., \& O’Brien, D. J. (1996). The use of the ces-d for measuring symptoms of depression in three rural russian villages. Social Indicators Research, 39(1), 89-108. https : //doi.org/10.1007/BF00300834

Faris, R. E. L., \& Dunham, H. W. (1939). Mental disorders in urban areas: An ecological study of schizophrenia and other psychoses.

Goldberg, D. P., Gater, R., Sartorius, N., Ustun, T. B., Piccinelli, M., Gureje, O., \& Rutter, C. (1997). The validity of two versions of the GHQ in the WHO study of mental illness in general health care. Psychological Medicine, 27(1), 191-197. https://doi.org/10. 1017/S0033291796004242

Hayes, A. F. (2013). An introduction to mediation, moderation, and conditional process analysis: A regression-based approach. Guilford Press.

Hundley, G., \& Lambie, G. (2007). Russian speaking immigrants from the commonwealth of independent states in the united states: Implications for mental health counselors. Journal of Mental Health Counseling, 29(3), 242-258. https://doi.org/10.17744/ mehc.29.3.34016u53586q4016

IBM Corp. (2011). IBM SPSS Statistics for Windows (Version 20.0). Armonk, NY: Author.

Jones, C. J., \& Trickett, E. J. (2005). Immigrant adolescents behaving as culture brokers: A study of families from the former soviet union. The Journal of Social Psychology, 145(4), 405-428. https://doi.org/10. 3200/SOCP.145.4.405-428

Juang, L. P., \& Alvarez, A. N. (2011). Family, school, and neighborhood: Links to chinese american adolescent perceptions of racial/ethnic discrimination. Asian American Journal of Psychology, 2(1), 1. https://doi.org/10.1037/a0023107

Jurcik, T., Ahmed, R., Yakobov, E., Solopieieva-Jurcikova, I., \& Ryder, A. G. (2013). Understanding the role of the ethnic density effect: Issues of acculturation, discrimination and social support. Journal of Community Psychology, 41(6), 662-678.

Jurcik, T., Chentsova-Dutton, Y. E., Solopieieva-Jurcikova, I., \& Ryder, A. G. (2013). Russians in treatment: The evidence base supporting cultural adaptations. Journal of Clinical Psychology, 69(7), 774-791.

Kennedy, M. A., Parhar, K. K., Samra, J., \& Gorzalka, B. (2005). Suicide ideation in different generations of immigrants. The Canadian Journal of Psychiatry, 50(6), 353-356.

Kozulin, A., \& Venge, A. (1995). Immigration without adaptation: The psychological world of russian immigrants in israel. Journal of Russian $\mathcal{F}$ East European Psychology, 33(2), 26-38. https://doi.org/10. 2753/RPO1061-0405330226
Kwag, K. H., Jang, Y., \& Chiriboga, D. A. (2012). Acculturation and depressive symptoms in hispanic older adults: Does perceived ethnic density moderate their relationship? Journal of Immigrant and Minority Health, 14(6), 1107-1111. https://doi.org/ 10.1007/s10903-015-0200-6

Landrine, H., \& Klonoff, E. A. (2004). Culture change and ethnic-minority health behavior: An operant theory of acculturation. Journal of Behavioral Medicine, 27(6), 527-555. https://doi.org/10.1007/s10865004-0002-0

Landry, R., \& Bourhis, R. Y. (1997). Linguistic landscape and ethnolinguistic vitality: An empirical study. Journal of Language and Social Psychology, 16(1), 23-49. https : / / doi . org / 10 . 1177 / $0261927 \times 970161002$

McCubbin, H. I., Holson, D. H., \& Larson, A. S. (1996). Family assessment: Resiliency, coping, adaptation: Inventories for research and practice. In H. McCubbin, I. Hamilton, A. Thompson, \& M. McCubbin (Eds.), F-copes: Family crisis oriented persona evaluation scales. (pp. 455-507). University of Wisconsin Publishers.

Miller, A. M., Birman, D., Zenk, S., Wang, E., Sorokin, O., \& Connor, J. (2009). Neighborhood immigrant concentration, acculturation, and cultural alienation in former soviet immigrant women. Journal of Community Psychology, 37(1), 88-105. https://doi.org/ 10.1002/jcop.20272

Mirsky, J. (2009). Mental health implications of migration. Social Psychiatry and Psychiatric Epidemiology, 44(3), 179. https://doi.org/10.1007/s00127-0080430- 1

Noh, S., \& Kaspar, V. (2003). Perceived discrimination and depression: Moderating effects of coping, acculturation, and ethnic support. American Journal of Public Health, 93(2), 232-238. https://doi.org/10.2105/ AJPH.93.2.232

Norenzayan, A., \& Heine, S. J. (2005). Psychological universals: What are they and how can we know? Psychological Bulletin, 131(5), 763. https://doi.org/10. 1037/0033-2909.131.5.763

Oishi, S., Kesebir, S., \& Snyder, B. H. (2009). Sociology: A lost connection in social psychology. Personality and Social Psychology Review, 13(4), 334-353. https://doi.org/doi:10.1177/1088868309347835

Persky, I., \& Birman, D. (2005). Ethnic identity in acculturation research: A study of multiple identities of jewish refugees from the former soviet union. Journal of Cross-Cultural Psychology, 36(5), 557-572. https://doi.org/10.1177/0022022105278542

Ponizovsky, A. M., Geraisy, N., Shoshan, E., Kremer, I., Smetannikov, E., \& Grinshpoon, A. (2007). Emo- 
tional distress among first-time patients attending outpatient mental health clinics in israel: An arabjewish comparative study. Israel Journal of Psychiatry and Related Sciences, 44(1), 62.

Preacher, K. J., \& Hayes, A. F. (2008). Asymptotic and resampling strategies for assessing and comparing indirect effects in multiple mediator models. Behavior Research Methods, 40(3), 879-891. https://doi.org/ 10.3758/BRM.40.3.879

Radloff, L. S. (1977). The CES-D scale: A self-report depression scale for research in the general population. Applied Psychological Measurement, 1(3), 385401. https://doi.org/10.1177/014662167700100306

Redfield, R., Linton, R., \& Herskovits, M. J. (1936). A memorandum for the study of acculturation. American Anthropologist, 38(1), 149-152. https://doi.org/10. 2307/2791001

Ritsner, M., Ponizovsky, A., \& Ginath, Y. (1997). Changing patterns of distress during the adjustment of recent immigrants: A 1-year follow-up study. Acta Psychiatrica Scandinavica, 95(6), 494-499. https://doi . org/https://doi.org/10.1111/j.1600-0447.1997. tb10137.x

Rousseau, C., Pottie, K., Thombs, B., Munoz, M., \& Jurcik, T. (2011). Post traumatic stress disorder: Evidence review for newly arriving immigrants and refugees. Canadian Medical Association Journal, 183, 1-11. https://doi.org/10.1503/cmaj.090313

Rudmin, F. (2009). Constructs, measurements and models of acculturation and acculturative stress. International Journal of Intercultural Relations, 33(2), 106-123. https://doi.org/10.1016/j.ijintrel.2008.12.001

Ryder, A. G., Alden, L. E., \& Paulhus, D. L. (2000). Is acculturation unidimensional or bidimensional? a headto-head comparison in the prediction of personality, self-identity, and adjustment. Journal of Personality and Social Psychology, 79(1), 49. https://doi.org/ 10.1037/0022-3514.79.1.49

Sam, D. L. (2006). Acculturation and health. In D. L. Sam \& J. W. Berry (Eds.), The cambridge handbook of acculturation psychology (pp. 452-468). Cambridge University Press.

Schwartz, S. J., Unger, J. B., Zamboanga, B. L., \& Szapocznik, J. (2010). Rethinking the concept of acculturation: Implications for theory and research. American Psychologist, 65(4), 237. https://doi.org/10. 1037/a0019330

Shaw, R. J., Atkin, K., Bécares, L., Albor, C. B., Stafford, M., Kiernan, K. E., Nazroo, J. Y., Wilkinson, R. G., \& Pickett, K. E. (2012). Impact of ethnic density on adult mental disorders: Narrative review. The British Journal of Psychiatry, 201(1), 11-19. https: //doi.org/10.1192/bjp.bp.110.083675

Singh-Manoux, A., Marmot, M. G., \& Adler, N. E. (2005). Does subjective social status predict health and change in health status better than objective status? Psychosomatic Medicine, 67(6), 855-861. https:// doi.org/10.1097/01.psy.0000188434.52941.a0

Stafford, M., Bécares, L., \& Nazroo, J. (2009). Objective and perceived ethnic density and health: Findings from a united kingdom general population survey. American Journal of Epidemiology, 170(4), 484493. https://doi.org/10.1093/aje/kwp160

Statistics Canada. (2006). 2006 census of population (Catalogue number 97-555-XCB2006007 [Montréal, Code462]. Retrieved from http://www12. statcan. ca/censusrecensement/2006/dp-pd/index-eng.cfm.

Statistics Canada. (2011). 2011 census metropolitan area of montreal, quebec, 2011 census of canada (Catalogue number 98-310-XWE2011004). Retrieved from http : / / www12 . statcan . gc . ca / census recensement / 2011/as - sa/fogs - spg/Facts - cma eng.cfm? $\mathrm{LANG}=$ Eng \&GK=CMA\& $\mathrm{GC}=462$.

Syed, M., \& Juan, M. J. D. (2012). Discrimination and psychological distress: Examining the moderating role of social context in a nationally representative sample of asian american adults. Asian American Journal of Psychology, 3(2), 104. https://doi .org/10. 1037/a0025275

Trickett, E., Persky, I., \& Espino, S. (2009). Acculturation research: Proxies as sources of concept obfuscation. In A. Gari \& K. Mylonas (Eds.), Quot erat demostrandum: From herodotus' ethnographic journeys to cross-cultural research. International Association for Cross-Cultural Psychology.

Whitley, R., Prince, M., McKenzie, K., \& Stewart, R. (2006). Exploring the ethnic density effect: A qualitative study of a london electoral ward. International Journal of Social Psychiatry, 52(4), 376-391. https: //doi.org/10.1177/0020764006067239

Zhang, J., Mandl, H., \& Wang, E. (2010). Personality, acculturation, and psychosocial adjustment of chinese international students in germany. Psychological Reports, 107(2), 511-525. https://doi.org/10.2466/07. 09.11.17.PR0.107.5.511-525

Zimet, G. D., Dahlem, N. W., Zimet, S. G., \& Farley, G. K. (1988). The multidimensional scale of perceived social support. Journal of Personality Assessment, 52(1), 30-41. https://doi.org/10.1207/ s15327752jpa5201_2 